\title{
Earthquake hydrology
}

Francesco La Vigna

ROMA CAPITALE Dip.10 Tutela ambientale e del verde - Protezione Civile

Uff. Geologia ed Idrogeologia Ambientale - Roma

francesco.lavigna@comune.roma.it

L'interazione tra le onde sismiche e le acque sotterranee è stata documentata da oltre 2000 anni (Manga, 2001). Ad esempio, diversi giorni prima del terremoto di Nankaido in Giappone, nel 1946 (M 8.3), il livello di acqua in alcuni pozzi è sceso di oltre $1 \mathrm{~m}$, e alcuni pozzi si sono prosciugati (Sato, 1978; Linde e Sacks, 2002). Non mancano esempi storici anche in Italia. Subito dopo il terremoto dell'Irpinia ( $\mathrm{Mw}$ 6.9) del 23 novembre 1980, la portata della sorgente Sanità di Caposele aumentò con valori senza precedenti (Celico, 1981). Il terremoto del Fucino del 13 Gennaio 1915 (Oddone, 1915) e il terremoto di Casamicciola del 28 luglio 1883 (Molin et al, 2003) mostrarono chiaramente anche prima dell'avvento dei moderni strumenti di misura, che le acque sotterranee e i terremoti sono collegati.

La spiegazione dei comportamenti delle acque sotterranee al verificarsi dei terremoti dovrebbe, in linea di principio, essere semplice perché riflette le deformazioni stesse che sono causate dalle onde sismiche. I terremoti cambiano lo stress statico (cioè, il movimento di due porzioni di crosta terrestre lungo una faglia genera una variazione dello stato di stress crostale che si era accumulato fino a quel momento) e provocano sollecitazioni dinamiche (a causa del passaggio delle onde sismiche). Entrambi gli stress aumentano con l'aumentare del momento sismico, ma decrescono in modo inverso con la distanza (Manga e Wang, 2007). In letteratura sono state definite tre categorie di distanza per spiegare gli effetti idrologici dovuti ai terremoti: il campo prossimale (near-field) è l'area intorno all'ipocentro a un raggio di distanza di circa 1 lunghezza della faglia sismogenetica, il campo intermedio (intermediate-field) è l'area compresa in un raggio maggiore di 1 lunghezza della faglia sismogenetica dall'ipocentro, ma entro un raggio di 10 lunghezze, e il campo distale (far-field) è più di 10 lunghezze dall'ipocentro (Wang \& Manga, 2010).

Nel campo distale, la sollecitazione dinamica di picco è di gran lunga maggiore rispetto alla variazione dello stress statico, mentre entrambe le sollecitazioni sono comparabili nel campo prossimale. Nel campo intermedio entrambe le sollecitazioni sono misurabili, ma la sollecitazione dinamica è maggiore di quella statica(Wang \& Manga, 2010). Come descritto le onde sismiche causano variazioni spaziali di stress e, quindi anche variazioni spaziali nella pressione di poro; la conseguente differenza di pressione dei pori genera dei gradienti di pressione e provoca flussi di fluidi (Manga \& Wang, 2007).

A seconda delle litologie coinvolte, le variazioni di pressione interstiziale e di stress cambiano anche le geometrie dei pori e lo stato di fatturazione (Manga \& Wang, 2007). Nei depositi incoerenti, la deformazione di taglio provoca il riassestamento dei granuli di sedimento tendendo a riempire i pori preesistenti. Questo effetto riduce l'originale porosità, nel fenomeno che viene comunemente definito consolidamento o compattazione. Di contro, la deformazione di taglio in depositi addensati provoca il rotolamento dei granuli di sedimento uno sopra l'altro tendendo a creare nuovi spazi vuoti. Questo processo aumenta il volume totale dei sedimenti ed è comunemente definito dilatanza. Il comportamento delle rocce litoidi è nettamente diverso e supera il suo limite elastico; la rottura di rocce consolidate provoca l'apertura di microfessure (Brace et al, 1966).

Diversi modelli sono stati utilizzati per interpretare i cambiamenti idrogeologici indotti dai terremoti. Holzer e Yound (2007) hanno proposto la consolidazione non drenata (compattazione) per spiegare i cambiamenti di pressione di poro nel near-field, e molti autori hanno suggerito che le onde sismiche possono aumentare la permeabilità delle rocce rimuovendo il riempimento delle fratture; questo fenomeno può portare alla ridistribuzione della pressione di poro e quindi a cambiamenti del campo piezometrico (Brodsky et al, 2003; Matsumoto et al, 2003; Wang e Chia, 2008). Muir-Wood e King (1993) infine hanno suggerito che le risposte idrogeologiche co-sismiche e post-sismiche possono essere funzione dei meccanismi focali del terremoto di attivazione.

Vi sono aree che risultano particolarmente sensibili da un punto di vista idrogeologico agli eventi sismici, anche se molto distanti dall'epicentro, e spesso queste aree coincidono con zone caratterizzate da tettonica attiva e da manifestazioni idrotermali, o caratterizzate da risalita di acque e/o gas profondi.

Recenti eventi sismici avvenuti sul territorio italiano sono stati studiati anche da questo punto di vista. Il terremoto de L'Aquila del 6 aprile 2009 ( $\mathrm{Mw} \mathrm{6.3)} \mathrm{ha} \mathrm{permesso} \mathrm{di}$ valutare effetti sia nel near-field dove le sorgenti collocate ai piedi del massiccio del Gran Sasso d'Italia hanno registrato delle variazioni in portata (Adinolfi Falcone et al, 2012), che nell'intermediate-field, nel bacino idrotermale delle Acque Albule (Tivoli-Guidonia, RM) a circa $65 \mathrm{~km}$ di distanza dall'area epicentrale, dove si sono registrate variazioni di livello della falda (Fig. 1) che sono state più brusche presso i laghi delle sorgenti termali (La Vigna et al, 2012). Anche la sequenza sismica dell'Emilia di maggio-giugno 2012 ha fatto registrare diversi effetti nelle acque sotterranee, sia molto evidenti, come le eruzioni di pozzi e la genesi di vulcanetti di fango, sia strumentali, come la variazione di livello in pozzi e piezometri (Marcaccio e Martinelli, 2012). 
Se in Italia esistesse, come in altri luoghi tettonicamente attivi della Terra (Itaba et al, 2010), una rete di monitoraggio delle acque sotterranee specifica, per registrare le variazioni indotte dai terremoti nelle falde acquifere delle aree più sensibili, questo settore della ricerca italiana potrebbe sicuramente crescere e beneficiare di interessantissimi dati.

\section{PRE-SISMICO}

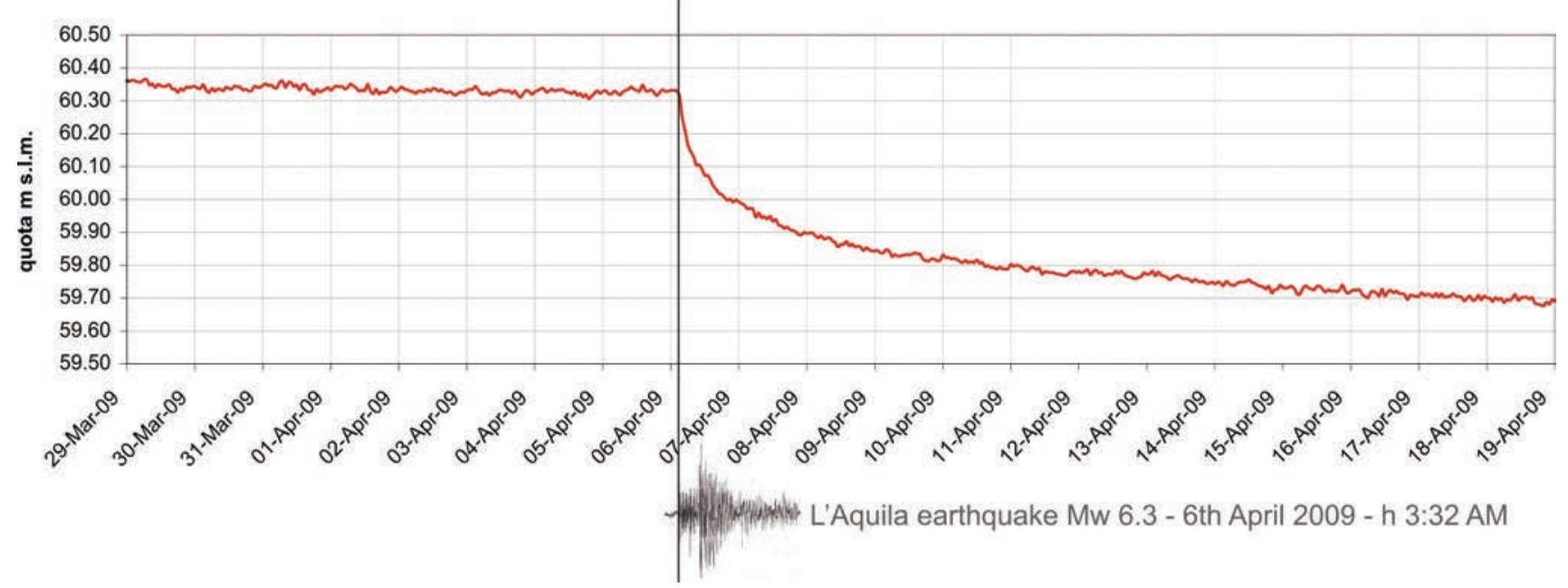

Fig.1: Variazioni di livello registrate negli acquiferi del bacino delle Acque Albule (RM) in corrispondenza del terremoto del 6 aprile 2009 a L'A quila (modificato da La Vigna et al, 2012).

\section{BIBLIOGRAFIA}

Adinolfi Falcone R., Carucci V., Falgiani A., Manetta M., Parisse B., Petitta M., Rusi S., Spizzico M., Tallini M. (2012) Changes on groundwater flow and hydrochemistry of the Gran Sasso carbonate aquifer due to the 2009 L'Aquila earthquake. Ital. J. Geosci. (Boll. Soc. Geol. It.), 131, 459-474.

Brace W.F., Paulding J. \& Scholz C. (1966) - Dilatancy in the fracture of crystalline rocks. J. Geophys. Res., 71, 3939-3953.

Brodsky E.E., Roeloffs E., Woodcock D., Gall I., Manga M. (2003) A mechanism for sustained groundwater pressure changes induced by distant earthquake. J. Geophys. Res., 108, 2390. doi: 10.1029/2002jb002321.

Celico P. (1981) Relazione tra idrodinamica sotterranea e terremoti in Irpinia (Campania). Rend. Soc. Geol. It., 4, 103-108.

Holzer T.L. \& Yound T.L. (2007) Liquefation, ground oscillation, and soil deformation at the wildlife array, California. Bull. Seism. Soc. Am., 97, 961-976.

Itaba S., Koizumi N., Matsumoto N., Othani R. (2010) Continuous Observation of Groundwater and Crustal Deformation for Forecasting Tonankai and Nankai Earthquakes in Japan. Pur. and Appl. Geophys., 167,1105-1114.

La Vigna F., Carucci V., Mariani I., Minelli L., Pascale F., Mattei M., Mazza R., Tallini M. (2012) Intermediate-field hydrogeological response induced by L'Aquila earthquake: the Acque Albule hydrothermal system (Central Italy). Ital. J. Geosci. (Boll. Soc. Geol. It.), 131, 475-485.

Linde AT., Sacks IS. (2002) Slow earthquakes and great earthquakes along the Nankai trough. Earth and Planet. Sci. Lett., 203, 265-75.

Manga M. (2001) Origin of postseismic streamflow change inferred from baseflow recession and magnitude-distance relations. Geophys. Res. Lett., 31 (10), 2133-2136.

\section{POST-SISMICO}

Manga M., Wang C.Y. (2007) Earthquake hydrology. In: KANAMORI H. (ed.), Treatise on geophysics, 4, Earthquake Seismology. Elsevier, 293-320.

Marcaccio M., Martinelli G. (2012) Effects on the groundwater levels of the May-June 2012 Emilia seismic sequence. ANNALS OF GEOPHYSICS, 55, 4, 2012; doi: 10.4401/ag-6139

Matsumoto N., Kitagawa G., Roeloffs E.A. (2003) Hydrological response to earthquake in the Haibara well, Central Japan. I. Water level changes revealed using state space decomposition of atmospheric pressure, rainfall and tidal responses, Geophys. J. Int., 155, 885-898.

Muir-Wood R., King G.C.P. (1993) Hydrological signatures of earthquake strain. J. Geophys. Res., 98 (B12), 20.035-20.068.

Molin P., Acocella V., Funiciello R. (2003) Structural seismic and hydrothermal features at the border of an active intermittent block: Ischia island (Italy). J. Volc. Geoth. Res., 121, 65-81.

Oddone E. (1915) Gli elementi fisici del grande terremoto marsicano fucense del 13 gennaio 1915. Boll. Soc. Sism. It., 19, 71-217.

Sato H. (1978) Precursory land tilt prior to the Tonankai earthquake of 1944. In some precursors prior to the recent great earthquakes along the Nankai trough. J. of Phys. of the Earth, 25, s11521.

Wang C.Y., Chia Y. (2008) - Mechanisms of water level changes during earthquakes: near-field versus intermediate-field. Geophys. Res. Lett., 35. doi: 10.1029/2008GL034227.

Wang C.Y., Manga M. (2010) - Earthquakes and Water. SpringerVerlag, 218. 